\title{
Incoherent spatial impulse response in variable-cross- section photoreceptors and frequency-domain analysis
}

\author{
Vasudevan Lakshminarayanan \\ School of Optometry and Department of Physics and Astronomy, University of \\ Missouri-St. Louis, 8001 Natural Bridge Road, St. Louis, Missouri 63121-4499 \\ Maria L. Calvo \\ Departamento de Optica, Facultad de Ciencias Físicas, Universidad Complutense, 28040 Madrid, Spain
}

Received February 10, 1995; revised manuscript received June 5, 1995; accepted June 22, 1995

\begin{abstract}
The spatial impulse response of a single variable-cross-section photoreceptor has been characterized assuming the incoming radiation to be an initial field propagating under confinement conditions inside the receptor. By application of Fourier analysis the total transfer function is determined. Both the outer and the inner segments are characterized as low-pass filters. The dependence of the transfer function on the modal parameter is analyzed. (c) 1995 Optical Society of America
\end{abstract}

\section{INTRODUCTION}

Since the pioneering work of Enoch in 1960, it has been experimentally established that retinal photoreceptors exhibit waveguiding properties. ${ }^{1}$ These studies have greatly added to our knowledge of the optical properties of the visual system, for example, in giving a more detailed interpretation of the Stiles-Crawford effect. ${ }^{2,3}$ Modeling the behavior of single photoreceptors as the incident radiation interaction that propagates under confinement conditions inside the receptor has resulted in a large number of important papers during the past few years (see, for example, Ref. 3). These models are, in general, developed as an application of waveguide theory and are therefore within the context of electromagnetic theory. Specifically, the total power confined inside the receptor gives, as a first approximation, information on their efficiency in transmitting the confined energy. An approximate treatment for the initial field was first established by Pask and Barrell. ${ }^{4}$ Assuming the absence of scattering in the light-photoreceptor interaction and, furthermore, that all the energy is confined along the receptor, the initial field is expressed as a superposition of discrete bound modes. This procedure is applicable in a large variety of problems describing a waveguiding phenomenon if one wishes to describe analytically the initial field falling on the entrance pupil of the waveguide. Among these problems is the case of a lens-photoreceptor system, in which an incident luminous signal strikes the entrance face of the photoreceptor. Previously, we studied the exact field and derived a formulation similar to that given by Pask and Barrell. The receptor optical properties were considered to be close to that of the surrounding medium (a weakly guiding waveguide). ${ }^{5}$ In subsequent studies we reformulated the initial field and obtained the total fraction of power confined within an absorbing photoreceptor. ${ }^{6}$ The implications of such an analysis were studied by consideration of values of the extinction coefficient for a typical vertebrate photoreceptor. ${ }^{7}$
In that analysis we concluded that, for a single-mode receptor, the shape of the radial distribution for the density of energy is independent of the waveguide absorption properties. To ensure the validity of the approximate formulation for the initial field we also analyzed the uniqueness of the decomposition of the incoming and the total fields and arrived at a direct and explicit expression for the fraction of the confined power within the receptor. ${ }^{8}$ The complexity of the previous formulation can be simplified by assumption of a linear behavior in the light-photoreceptor interaction phenomenon.

It is well established that a system may be regarded as the action of an operator transforming any input function into a unique output function. If the system obeys the two basic properties of additivity and homogeneity, it is called a linear system. The output is then characterized as a superposition integral whose kernel is designated as the impulse response of the system. This response represents the response of the system to a unit impulse. ${ }^{9}$ As an immediate consequence (assuming spatial invariance), the Fourier-transform properties for the convolution operation between the input and the impulse response lead to a description of the phenomenon in the spatial-frequency domain by defining the transfer function as the Fourier transform of the impulse response. In this domain the behavior of the system can be interpreted as a spatial-frequency filtering process. Keeping in mind the properties of spatially invariant linear systems and suitably performing operations in the integral expression representing the modal field, we can define a superposition integral whose kernel represents the action of the system. Thus we can establish an analogy between the behavior of the modal field striking the aperture plane of the receptor and the response of an optical system operating under linearity conditions. ${ }^{10}$ Analyzing the analytical expression of the kernel leads to a possible definition of a spatial impulse response for a space-variant system. In such an analysis the variance or the lack of reciprocity between the input and the output 
can be interpreted as being due to the presence of optical inhomogeneities in the photoreceptor structure. If we assume that they are homogeneous to a first approximation, then we can take an average refractive-index distribution as being close to a step function with radial symmetry (see Ref. 11 for experimental data). In such a situation, the photoreceptor would act as an invariant linear system. The spatial impulse response is related to the capability of the entrance pupil in diffracting the incident light and gives information on the modal field distribution for each propagation mode. The response can also be generalized to the case of incoherent illumination by definition of an impulse response as the squared modulus of the coherent response. We assumed a simple cylindrical geometry for the photoreceptor. Nevertheless, in reality photoreceptors exhibit a much more complex geometry and have to be considered as waveguides with variable cross section (tapered). Here we introduce a generalization for the initial field in a photoreceptor with inner-outer segment structure (for simplicity we do not consider the ellipsoid). We present this analysis in Section 2. The formulation is completed in Section 3, in which the boundary conditions are defined.

Applying the analogies with a cascade linear system, we define in Section 4 an approximate expression for the incoherent impulse response of both the inner and the outer segments. Application of the Fourier transform to these results gives the corresponding transfer function. The numerical analysis (Section 5) contains some results concerning these two functions. These results allow us to conjecture on the behavior of the single-mode photoreceptor as a low-pass filter (at least in the spatial-frequency bandwidth associated with the spatial-frequency resolution in the visual system of vertebrates ${ }^{12}$ ). However, small differences are noted between the outer and the inner segments. We compare the results with previous studies in which variable cross section and incoherent illumination were not considered. ${ }^{13}$ We end the paper with a brief conclusions section.

\section{CHARACTERIZATION OF THE INITIAL FIELD FOR THE CASE OF A PHOTORECEPTOR WITH VARIABLE CROSS SECTION}

As a general description let us consider an incoming electromagnetic field $\Psi_{\text {in }}(\mathbf{x})$ under the approximation of a scalar field striking the entrance face of a waveguide and propagating inside it. The waveguide is assumed to have a cylindrical geometry with some arbitrary periodicity along $z$. The partial differential equation for the field confined inside the receptor has a solution in the form of a propagation mode $^{5}$ :

$$
\begin{aligned}
\Phi(\mathbf{x}) & =\exp (i \beta z) \Psi_{x}(\mathbf{x}, z) \\
\mathbf{x} & \equiv(x, y, z)=(\mathbf{x}, z)
\end{aligned}
$$

where $\beta$ is a real value representing the propagation constant inside the waveguide and $\alpha$ is the modal order. $\Psi_{\alpha}(\mathbf{x}, z)$ is finite for any $(\mathbf{x}, z)$ and vanishes exponentially as $|x| \rightarrow+\infty$ for a given $z$ and is periodic in $z$. Furthermore, we assume that $\Psi_{\alpha}(\mathbf{x}, z)$ for fixed $z$ and that fixed $\alpha$ is practically constant inside the waveguide.
Based on this assumption and with the additional condition that the dielectric permittivity $\varepsilon(\mathbf{x})$ is equal to $\varepsilon_{c}$ (constant) outside the waveguide, $\Psi_{\alpha}(\mathbf{x}, z)$ satisfies the integral equation

$$
\begin{aligned}
\Psi_{\alpha}(\mathbf{x}, z)= & \Psi_{\text {in }}\left(\mathbf{x}, z_{0}\right)+\int_{0}^{z_{0}} \mathrm{~d} z^{\prime} \int_{\Omega} \mathrm{d}^{2} \mathbf{x}^{\prime} G\left(\mathbf{x}-\mathbf{x}^{\prime}, z-z^{\prime}\right) \\
& \times k^{2}\left[\varepsilon(\mathbf{x})-\varepsilon_{c}\right] \Psi_{\alpha}\left(\mathbf{x}^{\prime}, z\right) .
\end{aligned}
$$

In Eq. (2), $k=2 \pi / \lambda$ and is the wave vector, with $\lambda$ being the wavelength of the incident light. $z_{0}$ represents the finite-longitudinal dimension of the waveguide. $G\left(\mathbf{x}-\mathbf{x}^{\prime}, z-z^{\prime}\right)$ is a Green's function ${ }^{5}$ satisfying the partial differential equation as a solution in the form of a punctal source diverging from the origin: $\delta^{(2)}(\mathbf{x}) \delta(z)$. Also, $\mathbf{x}$ and $\mathbf{x}^{\prime}$ are both two-dimensional vector positions lying inside the aperture. $\Omega$ is the finite cross section of the waveguide. Note that $\varepsilon(\mathbf{x})-\varepsilon_{c}=0$ for points outside $\Omega$. Equation (2) is equivalent to the scalar differential wave equation, along with the boundary conditions determined by $\Psi_{\text {in }}(\mathbf{x})$.

This general formulation is applicable to all problems involving confinement of electromagnetic radiation. In the present study it is assumed that the retinal photoreceptors behave like waveguides exhibiting a specific cylindrical geometry, and one can establish the formulation given below.

A simple analytic treatment is the analysis of Eq. (2) for a fixed $\alpha$ value, where $\Omega$ is the constant cross section as noted above and $\varepsilon(\mathbf{x})$ is the dielectric permittivity function that varies for any $\mathbf{x}$ in the case of an inhomogeneous receptor.

However, in real life, photoreceptors have a nonuniform or variable cross section: a constant cross section $\Omega_{i}$ for the inner segment, a gradually variable cross section in the ellipsoid $\Omega(z)$, and a constant cross section $\Omega_{o}$ for the outer segment (with the area of $\Omega_{o}$ being smaller than $\Omega_{i}$ ), as shown in Fig. 1 . Here $z$ is the axial direction of the photoreceptor. To describe the effects of these different cross sections in the initial field, it is reasonable to consider, as a first step, only $\Omega_{i}$ and $\Omega_{o}$, omitting in the mathematical formulation the effect of the gradual cross section $\Omega(z)$ associated with the ellipsoid, which would greatly increase the complexity of the problem.

Thus let us assume the case of a photoreceptor as shown in Fig. 2. There is a direct connection between the inner and the outer segments exactly at the $z_{0}$ plane, and the entrance and the exit pupils of the photoreceptor are at $z=0$ and at $z=+\infty$, respectively.

In this situation the total field satisfies the following integral equation for $0<z<z_{0}$ and $z_{0}<z<+\infty$ :

$$
\begin{aligned}
\Psi(\mathbf{x}, z)= & \Psi_{\text {in }}(\mathbf{x}, z)+\int_{\Omega_{i}} \mathrm{~d}^{2} \mathbf{x}^{\prime} \int_{0}^{z_{0}} \mathrm{~d} z^{\prime} G\left(\mathbf{x}-\mathbf{x}^{\prime}, z-z^{\prime}\right) \\
& \times k^{2}\left[\varepsilon\left(\mathbf{x}^{\prime}\right)-\varepsilon_{c}\right] \cdot \Psi\left(\mathbf{x}^{\prime}, z\right) \\
& +\int_{\Omega_{o}} \mathrm{~d}^{2} \mathbf{x}^{\prime} \int_{z_{0}}^{+\infty} \mathrm{d} z^{\prime} G\left(\mathbf{x}-\mathbf{x}^{\prime}, z-z^{\prime}\right) \\
& \times k^{2}\left[\varepsilon\left(\mathbf{x}^{\prime}\right)-\varepsilon_{c}\right] \cdot \Psi\left(\mathbf{x}^{\prime}, z\right) .
\end{aligned}
$$

We can establish solutions for Eq. (3) that are superpositions of propagation modes for each segment without radiation, namely: 


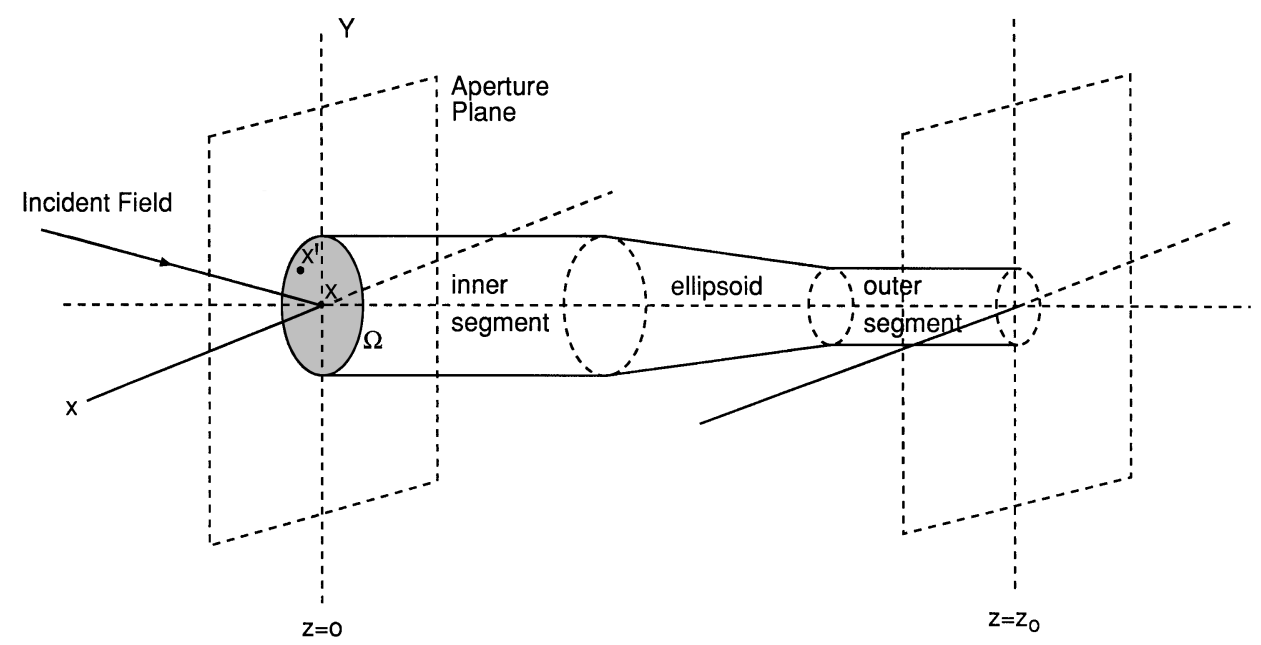

Fig. 1. Model for the aperture plane geometry in a photoreceptor.

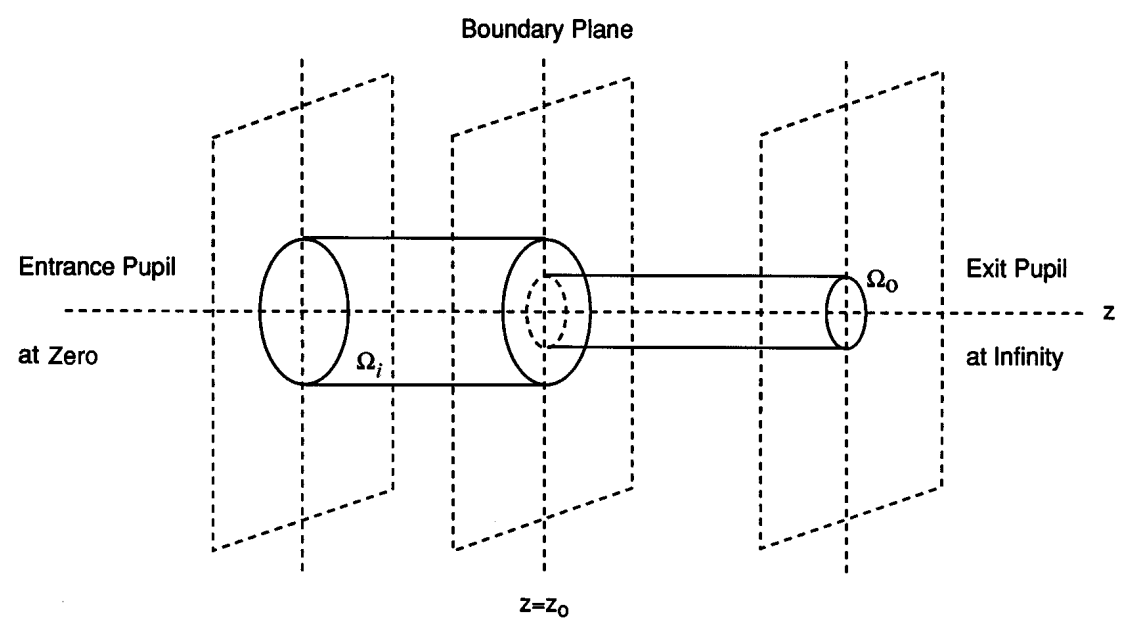

Fig. 2. Geometry for the inner-outer segment coupling.

$\Psi(\mathbf{x}, z)=\left\{\begin{array}{r}\sum_{\alpha} c_{\alpha, i} \exp \left(i \beta_{\alpha, i} z\right) \Phi_{\alpha, i}(\mathbf{x}) ; 0 \leq z \leq z_{0} \\ \text { inner segment } \\ \sum_{\alpha} c_{\alpha, o} \exp \left(i \beta_{\alpha, o} z\right) \Phi_{\alpha, o}(\mathbf{x}) ; z_{0} \leq z \leq+\infty \\ \text { outer segment }\end{array}\right.$

In Eq. (4), $\beta_{i, \alpha}$ and $\beta_{o, \alpha}$ are propagation constants for the inner and the outer segments, respectively. The subindex $\alpha$ denotes the modal order for each propagation mode. The sums in Eq. (4) are extended in principle to all the values of $\alpha, \beta_{i}$, and $\beta_{0}$, such that $k n_{c} \leq \beta_{\alpha, i} \leq k n_{i}$ and $k n_{c} \leq \beta_{\alpha, o} \leq k n_{o}$ (for a given mode). Here $n_{i}$ is the refractive index of the inner segment, and $n_{o}$ is the refractive index of the outer segment. $\phi_{\alpha, i}$ and $\phi_{\alpha, o}$ are the propagation modes for the inner and the outer segments and are given by Eq. (1).

Inside the inner and the outer segments several modes are present, as observed experimentally by Enoch. ${ }^{1}$ The superposition in Eq. (4) will be defined only for a certain number of terms. As a first approximation we assume a single-mode of zero order: $\alpha=0$, and consequently only one term of the series has to be considerd.

At this point notice that solutions for the modal fields $\Phi_{\alpha, i}$ and $\Phi_{\alpha, o}$ are equivalent to the following integral equation:

$$
\begin{aligned}
\Phi_{\alpha, i}(\mathbf{x})= & (-1 / 4 i) \int_{\Omega_{i}} \mathrm{~d}^{2} \mathbf{x}^{\prime} H_{0}^{(1)}\left(i \chi_{\alpha, i}\left|\mathbf{x}-\mathbf{x}^{\prime}\right|\right) \\
& \times k^{2}\left[\varepsilon\left(\mathbf{x}^{\prime}\right)-\varepsilon_{c}\right] \Phi_{\alpha, i}\left(\mathbf{x}^{\prime}\right), \\
\Phi_{\alpha, o}(\mathbf{x})= & (-1 / 4 i) \int_{\Omega_{o}} \mathrm{~d}^{2} \mathbf{x}^{\prime} H_{0}^{(1)}\left(i \chi_{\alpha, o}\left|\mathbf{x}-\mathbf{x}^{\prime}\right|\right) \\
& \times k^{2}\left[\varepsilon\left(\mathbf{x}^{\prime}\right)-\varepsilon_{c}\right] \Phi_{\alpha, o}\left(\mathbf{x}^{\prime}\right) .
\end{aligned}
$$

Here $H_{0}^{(1)}$ is the outgoing zero-order Hankel function of the first kind, which is found in all problems of propagation in which a cylindrical geometry is involved. It is exponentially damped for large $|\mathbf{x}|$, that is, for points inside each segment that are well separated, because it depends on $i \chi_{\alpha}\left|\mathbf{x}-\mathbf{x}^{\prime}\right|$ in both the inner and the outer segments. ${ }^{14}$ Also,

$$
\begin{aligned}
& \varepsilon_{c} k^{2}={\beta_{\alpha, i}}^{2}-\chi_{\alpha, i}{ }^{2}, \\
& \varepsilon_{c} k^{2}=\beta_{\alpha, o}{ }^{2}-\chi_{\alpha, o}{ }^{2} .
\end{aligned}
$$

The modal parameter associated with the inner segment (or the outer segment with subindex 0 ) that is real for a nonabsorbing structure is given by $\chi_{\alpha, i}$. The dielectric permittivity of the surrounding medium is given by $\varepsilon_{c} . \quad k$ is the propagation constant in free space. The largest value of $\chi_{\alpha, i}$ is $(w / c)\left(\varepsilon_{i}-\varepsilon_{c}\right)^{1 / 2}$, where $w$ is the an- 
gular frequency and $c$ is the speed of light. The modal parameter is proportional to the numerical aperture of each segment.

\section{BOUNDARY CONDITIONS}

We assume that the following two equations are satisfied for any $\mathbf{x}$ :

$$
\sum_{\alpha} c_{\alpha, i}\left[\exp \left(i \beta_{i} z_{0}\right)\right] \Phi_{\alpha, i}(\mathbf{x})=\sum_{\alpha} c_{\alpha, o}\left[\exp \left(i \beta_{o} z_{0}\right)\right] \Phi_{\alpha, o}(\mathbf{x}),
$$

$\sum_{\alpha} c_{\alpha, i} \beta_{i}\left[\exp \left(i \beta_{i} z_{0}\right)\right] \Phi_{\alpha, i}(\mathbf{x})=\sum_{\alpha} c_{\alpha, o} \beta_{o}\left[\exp \left(i \beta_{o} z_{0}\right)\right] \Phi_{\alpha, o}(\mathbf{x})$,

with $\phi_{\alpha, i}$ and $\phi_{\alpha, o}$ satisfying Eqs. (5) and (6).

By substituting Eq. (8) into Eq. (4) for both $0 \leq z \leq z_{0}$ and $z_{0} \leq z \leq+\infty$ one integrates first over $z$ and then over the dummy variable $\mathbf{l}_{z}$ [longitudinal wave vector in the $z$ direction (waveguide axis)] contained inside $G(\mathbf{x}-$ $\mathbf{x}^{\prime}, z-z^{\prime}$ ). Then, by using Eqs. (5) and (6), one can see that important cancellations take place. After these cancellations, one can obtain the general form of the initial field that gives rise to the total field. This expression is given by

$$
\begin{aligned}
\Psi_{\text {in }}(\mathbf{x}, z)= & -\sum_{\alpha} c_{\alpha, i} \cdot k^{2} \int_{\Omega_{i}} \mathrm{~d}^{2}\left(\mathbf{x}^{\prime}\right)\left[\varepsilon\left(\mathbf{x}^{\prime}\right)-\varepsilon_{c}\right] \\
& \times \Phi_{\alpha, i}\left(\mathbf{x}^{\prime}\right) \cdot\left[1 /(2 \pi)^{2}\right] \int \mathrm{d}^{2}(\mathbf{l}) \exp \left[i \mathbf{l}\left(\mathbf{x}-\mathbf{x}^{\prime}\right)\right] \\
& \cdot[1 / 2 \sigma(\mathbf{l})] \cdot\left\{1 /\left[\beta_{i}-\sigma(\mathbf{l})\right]\right\} \exp [i \sigma(\mathbf{l}) z] \\
& \cdot\left(\exp \left\{i z_{0}\left[\beta_{i}-\sigma(\mathbf{l})\right]\right\}-1\right)-\sum_{\alpha} c_{\alpha, o} \exp \left(i \beta_{o} z_{0}\right) \\
& \cdot k^{2} \int_{\Omega_{o}} \mathrm{~d}^{2}\left(\mathbf{x}^{\prime}\right)\left[\varepsilon\left(\mathbf{x}^{\prime}\right)-\varepsilon_{c}\right] \Phi_{\alpha, i}\left(\mathbf{x}^{\prime}\right) \cdot\left[1 /(2 \pi)^{2}\right] \\
& \cdot \int \mathrm{d}^{2} \mathbf{l} \cdot \exp \left[i\left(z-z_{0}\right) \sigma(\mathbf{l})\right] \cdot[1 / 2 \sigma(\mathbf{l})] \\
& \cdot\left\{1 /\left[\sigma(\mathbf{l})-\beta_{o}\right]\right\} \cdot \exp \left[i \mathbf{l}\left(\mathbf{x}-\mathbf{x}^{\prime}\right)\right] \\
= & \Psi_{\mathrm{in}, \geq}(\mathbf{x}, z),
\end{aligned}
$$

where $\sigma(\mathbf{l})=\left(k^{2}-\mathbf{l}^{2}\right)^{1 / 2}$.

For the region $0 \leq z \leq z_{0}$,

$$
\begin{aligned}
\Psi(\mathbf{x}, z)= & -\sum_{\alpha} c_{\alpha, i} k^{2} \int_{\Omega_{i}} \mathrm{~d}^{2}\left(\mathbf{x}^{\prime}\right)\left[\varepsilon\left(\mathbf{x}^{\prime}\right)-\varepsilon_{c}\right] \Phi_{\alpha, i}\left(\mathbf{x}^{\prime}\right) \\
& \cdot\left[1 /(2 \pi)^{2}\right] \int \mathrm{d}^{2} \mathbf{l} \exp \left[i \mathbf{l}\left(\mathbf{x}-\mathbf{x}^{\prime}\right)\right] \cdot[1 / 2 \sigma(\mathbf{l})] \\
& \times\left\{\frac{\exp [i z \sigma(\mathbf{l})]}{\sigma(\mathbf{l})-\beta_{i}}+\frac{\exp \left(i z_{0} \beta_{i}\right) \exp \left[i\left(z_{0}-z\right) \sigma(\mathbf{l})\right]}{\sigma(\mathbf{l})+\beta_{i}}\right\} \\
& +\sum_{\alpha} c_{\alpha, o} k^{2} \exp \left(i z_{0} \beta_{o}\right) \int_{\Omega_{o}} \mathrm{~d}^{2} \mathbf{x}^{\prime}\left[\varepsilon\left(\mathbf{x}^{\prime}\right)-\varepsilon_{c}\right] \\
& \times \Phi_{\alpha, o}\left(\mathbf{x}^{\prime}\right) \cdot\left[1 /(2 \pi)^{2}\right] \int \mathrm{d}^{2} \mathbf{l} \exp \left[i \mathbf{l}\left(\mathbf{x}-\mathbf{x}^{\prime}\right)\right] \\
& \times \frac{\left.\exp \left[i\left(z-z_{0}\right) \mid-\sigma(\mathbf{l})\right]\right]}{2 \sigma(\mathbf{l})\left[\beta_{o}+\sigma(\mathbf{l})\right]} \\
= & \Psi_{\mathrm{in}, \leq}(\mathbf{x}, z) .
\end{aligned}
$$

For both Eqs. (10) and (11) the scalar differential equation

$$
\left(\nabla^{2}+k^{2}\right) \Psi_{\text {in }}=0
$$

holds.

It is easy to find the form of $\Psi_{\text {in }}$ for $z \leq 0$. Here $\Psi_{\text {in }}$ and its first derivative with respect to $z$ coincide at $z=0$ with $\Psi_{\mathrm{in}, \leq},\left(\partial \Psi_{\mathrm{in}, \leq}\right) / \partial z$, for any $x$. For brevity we omit details.

To prove the correctness of Eqs. (10) and (11), let us analyze the behavior of the initial field $\Psi_{\text {in }}$ at the $z_{0}$ plane. This function has to satisfy the boundary conditions

$$
\begin{gathered}
\Psi_{\mathrm{in}, \leq}\left(\mathbf{x}, z_{0}\right)=\Psi_{\mathrm{in}, \geq}\left(\mathbf{x}, z_{0}\right), \\
{\left[\frac{\partial \Psi_{\mathrm{in}, \leq}(\mathbf{x}, z)}{\partial z}\right]_{z=z_{0}}=\left[\frac{\partial \Psi_{\mathrm{in}, \geq}(\mathbf{x}, z)}{\partial z}\right]_{z=z_{0}} .}
\end{gathered}
$$

By substituting Eqs. (10) and (11) into Eqs. (12) and (13), canceling out terms, and using Eqs. (5) and (6) and then Eqs. (8) and (9), we can demonstrate that the boundary conditions given by Eqs. (12) and (13) are exactly satisfied.

\section{ANALOGIES BETWEEN THE MODAL FIELD AND THE RESPONSE IN A LINEAR SYSTEM}

By suitably performing operations on Eqs. (5) and (6) we can establish certain analogies between the behavior of the modal field defined above and the response of a linear system. This calculation leads to a simpler physical interpretation of the excitation field at the entrance pupil of the inner segment. Then the propagated signal interacts with the outer segment. We neglect scattering effects at the inner-outer segment interface and assume that mode propagation is only along the positive $z$ direction. Under linearity conditions a phenomenon similar to that which occurs in a cascade linear system takes place. We assume that the field after propagation along the inner segment behaves like the input field at the entrance face of the outer segment. Operating in this fashion, we have the mathematical framework to define the corresponding total transfer function as the product of the two transfer functions associated with the inner and the outer segments.

Let us denote the following formulation (without subscripts $i$ or $o$, for simplicity) and define similar expressions for each of the segments under consideration.

Because $\left[\varepsilon(\mathbf{x})-\varepsilon_{c}\right]$ is different from zero for points defined inside the aperture, by multiplying both sides of Eq. (5) or (6) by $\left[\varepsilon(\mathbf{x})-\varepsilon_{c}\right]^{1 / 2}$ we obtain

$$
\begin{aligned}
{\left[\varepsilon(\mathbf{x})-\varepsilon_{c}\right]^{1 / 2} \Phi_{\alpha}(\mathbf{x})=} & (-1 / 4 i) \int_{\Omega} \mathrm{d}^{2} \mathbf{x}^{\prime}\left\{\left[\varepsilon(\mathbf{x})-\varepsilon_{c}\right]^{1 / 2}\right. \\
& \left.\cdot H_{0}^{(1)}\left(i \chi_{\alpha}\left|\mathbf{x}-\mathbf{x}^{\prime}\right|\right) k^{2}\left[\varepsilon\left(\mathbf{x}^{\prime}\right)-\varepsilon_{c}\right]^{1 / 2}\right\} \\
& \times\left[\varepsilon\left(\mathbf{x}^{\prime}\right)-\varepsilon_{c}\right]^{1 / 2} \Phi_{\alpha}\left(\mathbf{x}^{\prime}\right)
\end{aligned}
$$

We define

$$
\begin{aligned}
K_{\alpha}\left(\mathbf{x}, \mathbf{x}^{\prime}\right)= & \left(-k^{2} / 4 i\right)\left\{\left[\varepsilon(\mathbf{x})-\varepsilon_{c}\right]^{1 / 2}\right. \\
& \left.\times H_{0}^{(1)}\left(i \chi_{\alpha}\left|\mathbf{x}-\mathbf{x}^{\prime}\right|\right)\left[\varepsilon\left(\mathbf{x}^{\prime}\right)-\varepsilon_{c}\right]^{1 / 2}\right\},
\end{aligned}
$$




$$
\begin{aligned}
& g(\mathbf{x})=\left[\varepsilon(\mathbf{x})-\varepsilon_{c}\right]^{1 / 2} \Phi_{\alpha}(\mathbf{x}), \\
& g\left(\mathbf{x}^{\prime}\right)=\left[\varepsilon\left(\mathbf{x}^{\prime}\right)-\varepsilon_{c}\right]^{1 / 2} \Phi_{\alpha}\left(\mathbf{x}^{\prime}\right) .
\end{aligned}
$$

If we substitute Eqs. (15) and (16) into Eq. (14), the integral equation characterizing the bound states associated with the propagation modes becomes, for all points inside the aperture,

$$
g(\mathbf{x})=\int_{\Omega} \mathrm{d}^{2} \mathbf{x}^{\prime} K_{\alpha}\left(\mathbf{x}, \mathbf{x}^{\prime}\right) g\left(\mathbf{x}^{\prime}\right) .
$$

The equation given above has a form similar to that describing the response of a space-variant linear optical system, ${ }^{15}$ with the particular feature that it is limited to the radial dimensions of the segment. Equation (17) is equivalent to the so-called superposition integral, and therefore the kernel $K_{\alpha}\left(\mathbf{x}, \mathbf{x}^{\prime}\right)$ of this integral behaves like a transverse impulse response associated with the diffraction capability of the entrance pupil of each segment. ${ }^{9}$ The form of the kernel indicates that this impulse response depends on both where the impulse was applied $(\mathbf{x})$ and where one observes the output $\left(\mathbf{x}^{\prime}\right)$. Consequently, for each propagation mode, we have the corresponding impulse response as given by Eq. (15). The formulation also indicates that the photoreceptor would have a limited capability for transmitting and processing the incident energy, with the limitations being related to the radial dimensions and the optical properties of each structure.

Equation (17) can be simplified as follows. As discussed by Horowitz, ${ }^{16}$ individual segments could be assumed to be homogeneous. This is clearly a first-order approximation inasmuch as each photoreceptor has a complex structure with local inhomogeneities. We use the measured average values of the refractive index of the cone inner segment and the cone outer segment (1.39 and 1.385 , respectively) that were reported by Sidman. ${ }^{17}$ If we assume a refractive-index gradient, this would influence the form of the kernel $K_{\alpha}\left(\mathbf{x}, \mathbf{x}^{\prime}\right) .{ }^{18}$ According to Eq. (15),

$$
\begin{aligned}
K_{\alpha}\left(\mathbf{x}, \mathbf{x}^{\prime}\right) & =\left(-k^{2} / 4 i\right)\left(\varepsilon-\varepsilon_{c}\right) H_{0}^{(1)}\left(i \chi_{\alpha}\left|\mathbf{x}-\mathbf{x}^{\prime}\right|\right) \\
& =K\left(\mathbf{x}-\mathbf{x}^{\prime}\right)
\end{aligned}
$$

Consequently Eq. (17) becomes

$$
g(\mathbf{x})=\int_{\Omega} \mathrm{d}^{2} \mathbf{x}^{\prime} K_{\alpha}\left(\mathbf{x}-\mathbf{x}^{\prime}\right) g\left(\mathbf{x}^{\prime}\right) .
$$

Equation (19) represents a new form of the superposition integral, wherein the system behaves like a spatially invariant system, with the invariance being due to the dependence of the kernel $K_{\alpha}\left(\mathbf{x}-\mathbf{x}^{\prime}\right)$ on the difference of spatial coordinates $\left(\mathbf{x}-\mathbf{x}^{\prime}\right)$. The superposition integral is now a convolution operation between $K_{\alpha}(\mathbf{x})$ and $g(\mathbf{x})$. Because the integral in Eq. (19) is zero for all points outside the aperture, we can extend the integration limits to infinity and can write

$$
g(\mathbf{x})=\int_{-\infty}^{+\infty} \mathrm{d}^{2}\left(\mathbf{x}^{\prime}\right) K_{\alpha}\left(\mathbf{x}-\mathbf{x}^{\prime}\right) g\left(\mathbf{x}^{\prime}\right) p\left(\mathbf{x}^{\prime}\right),
$$

where $p\left(\mathbf{x}^{\prime}\right)$ is the pupil function associated with each segment. We can also write Eq. (20) as

$$
g(\mathbf{x})=[g(\mathbf{x}) p(\mathbf{x})] * K_{\alpha}(\mathbf{x}) .
$$

In the above equation the symbol $*$ denotes convolution.
From Eq. (21) the integral equation corresponding to the bound states would be analogous to the response of a transversally space-invariant linear system. The behavior of the kernel $K_{\alpha}\left(\mathbf{x}-\mathbf{x}^{\prime}\right)$ of the integral equation, which represents the impulse response function, is simply that of a zero-order Hankel function of the first kind [see Eq. (18)] with a pure imaginary argument. This behavior is affected by a constant defined in terms of the optical properties of the segment (e.g., numerical aperture). The argument of the Hankel function also depends on the values of the modal parameter $\chi_{\alpha}$.

Next we discuss the impulse response and transfer function of the incoherently illuminated photoreceptor.

According to Eq. (18), for the inner segment we define

$$
\left|K_{\alpha}\left(\mathbf{x}-\mathbf{x}^{\prime}\right)\right|_{i}^{2}=\left(k^{4} / 16\right)\left(\varepsilon_{i}-\varepsilon_{c}\right)\left|H_{0}^{(1)}\left(i \chi_{\alpha, i}\left|\mathbf{x}-\mathbf{x}^{\prime}\right|\right)\right|^{2}
$$

as the incoherent impulse response for this section of the photoreceptor. We can also write a similar expression:

$$
\left|K_{\alpha}\left(\mathbf{x}-\mathbf{x}^{\prime}\right)\right|_{o}^{2}=\left(k^{4} / 16\right)\left(\varepsilon_{o}-\varepsilon_{c}\right)\left|H_{0}^{(1)}\left(i \chi_{\alpha, o}\left|\mathbf{x}-\mathbf{x}^{\prime}\right|\right)\right|^{2},
$$

as the incoherent impulse response for the outer segment.

According to the properties of $H_{0}^{(1)}$, depending on an imaginary variable, we can write, in general ${ }^{14}$

$$
\left|H_{0}^{(1)}\left(i \chi_{\alpha} \mid \mathbf{x}-\mathbf{x}^{\prime}\right)\right|^{2}=\left(4 / \pi^{2}\right)\left|K_{0}\left(\chi_{\alpha}\left|\mathbf{x}-\mathbf{x}^{\prime}\right|\right)\right|^{2},
$$

where $K_{0}$ is a Kelvin function. The mathematical behavior of its square modulus is that of a damped function with a singularity at the origin. ${ }^{18}$

By suitable manipulations of Eq. (24) and by using the properties of a Kelvin function ${ }^{18}$ we obtain an approximate expression:

$$
\begin{aligned}
\left|H_{0}^{(1)}\left(i \chi_{\alpha}\left|\mathbf{x}-\mathbf{x}^{\prime}\right|\right)\right|^{2} & \approx\left(2 / \pi\left|\chi_{\alpha}\right| \cdot\left|\mathbf{x}-\mathbf{x}^{\prime}\right|\right) \\
\cdot & \exp \left(-2 \chi_{\alpha}\left|\mathbf{x}-\mathbf{x}^{\prime}\right|\right) \cdot \exp (-\pi / 2) .
\end{aligned}
$$

If relation (25) is substituted into Eqs. (22) and (23), we obtain the approximate formula for analyzing the incoherent impulse response for both the inner and the outer segments. We note that the damping term in relation (25) will be affected by the values of the modal parameter considered for each segment. The possible differences, which in any case can be expected to be small, will arise from the optical properties of each section, namely, dielectric permittivity values and numerical aperture for a fixed average wavelength.

Considering a linear behavior for the system, one would obtain the total impulse response of the photoreceptor, as defined for the present model, as a convolution of the impulse response for the inner segment [Eq. (22)] with that for the outer segment [Eq. (23)]. An important assumption, as noted above, is that the reflection of the propagated signal at the interface between the inner and the outer segments is ignored. According to the analytical expression for $\left|H_{0}^{(1)}\left(i \chi_{\alpha}\left|\mathbf{x}-\mathbf{x}^{\prime}\right|\right)\right|^{2}$ as given by relation (25), the operation is not trivial. A procedure for circumventing this difficulty would be the formulation of the problem in the spatial-frequency domain. Additionally, this 
procedure gives a method for analyzing the response of the photoreceptor in terms of spatial resolution. In this case the Fourier transform of the convolution operation is represented as the product of the transfer function associated with the inner segment times that for the outer segment. This product defines the total transfer function of the system. Operating in this fashion, for any particular arbitrary input $f(\mathbf{x})$, one can obtain the spectrum of the output as the product of the spectrum of $f(\mathbf{x})$ times the total transfer function of the system. In so doing, one could in principle decompose $f(\mathbf{x})$ as a sum of discrete bound modes and could operate for each mode. Because the transformation is linear, one should be able to reproduce the output as the discrete sum of each modal response. Under this interpretation, no coupling phenomena are considered.

Applying the Fourier transform in Eqs. (22) and (23) together with relation (25) leads to the corresponding transfer function for both segments. Then, by the reciprocal procedure, taking the inverse Fourier transform of the total optical transfer function will give the result for the total impulse response of the system.

Using this procedure, one calculates the transfer function of the inner segment to be

$$
\begin{aligned}
F\left(\chi_{i}, \rho\right)= & \left(k^{2} / 2\right)\left(1 / \chi_{i}\right) \exp (-\pi / 2)\left(\varepsilon_{i}-\varepsilon_{c}\right)^{2} \\
& \times\left[4 \chi_{i}^{2}+(2 \pi \rho)^{2}\right]^{-1} .
\end{aligned}
$$

In Eq. (26), $\rho$ is the radial spatial frequency associated with the spatial coordinate: $r=\left(\mathbf{x}-\mathbf{x}^{\prime}\right)^{1 / 2}$, and the properties of the Fourier transform have been used to derive the expression. ${ }^{19}$ An equation similar to Eq. (26) can be defined for the outer segment with subindex 0 .

Equation (26), along with an analogous expression for the outer segment, represents the transfer function associated with the inner-outer segments. At this point it should be realized that both analyses [Eqs. (22) and (23) and Eq. (26)] are equivalent. It is clear, nevertheless, that the results in the spatial-frequency domain will give more interesting conclusions regarding the photoreceptor's behavior as a signal filtering process as well as the percentage of attenuation of both low and high spatial frequencies. This idea is widely applied in vision research because of the implications of the response of the visual system to patterns. ${ }^{12}$ A similar analysis can be carried out for the case of coherent illumination. For this case the coherent impulse response is given by $K_{\alpha}$. Both functions could be useful under different conditions. Stacey and Pask, ${ }^{13}$ for example, introduced a coherent impulse response by assuming a sinusoidal illumination of the photoreceptor, which they claim is applicable to the interpretation of visual acuity tests. We suggest that both conditions should be considered. In a real-life situation, the visual system functions under incoherent illumination. The coherent case should be applicable to some specific tests, as discussed by Stacey and Pask. ${ }^{13}$

\section{NUMERICAL RESULTS}

We numerically analyzed Eqs. (22) and (23), together with Eq. (26). The physical parameters were taken from published experimental studies. ${ }^{17,20}$ The average diameter of the inner segment is taken to be approximately $2.0 \mu \mathrm{m}$, with an average refractive index of 1.353; the outer segment (with calyx) has an average diameter of $1.55 \mu \mathrm{m}$ and an average refractive index of 1.43 ; and the surrounding extracellular matrix has a refractive index of 1.34. We calculate the modal-parameter values by considering the behavior of the photoreceptor to be that of a single-mode waveguide supporting the set of fundamental modes. We recall that the modal parameters are defined in terms of the radius of the waveguide, the refractive index, and the propagation constants $k$ and $\beta .^{21}$

The modal parameter is determined through the cutoff condition for a set of fundamental modes $(\alpha=0)$ :

$$
V_{0}^{2}=U_{0}^{2}+W_{0}^{2}
$$

with $V_{0}=2.405$, where

$$
\begin{gathered}
U_{0}=\chi_{0} \cdot R, \quad W_{0}=\chi_{0}{ }^{\prime} \cdot R, \\
U_{0}=R\left(k^{2} n^{2}-\beta_{0}{ }^{2}\right)^{1 / 2},
\end{gathered}
$$

where $R$ is the radius of the waveguide; $k=2 \pi / \lambda$, with $\lambda$ being the wavelength of the incident light; $n$ is the

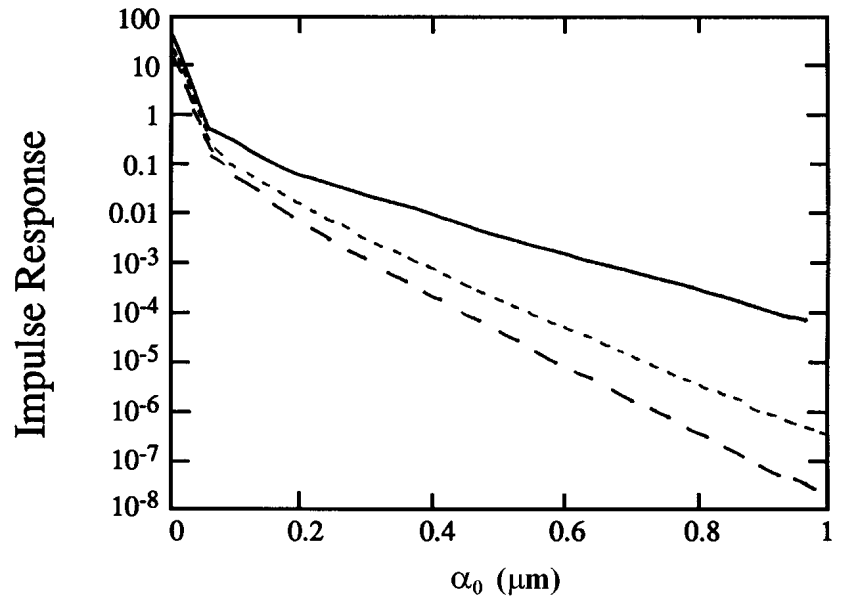

(a)

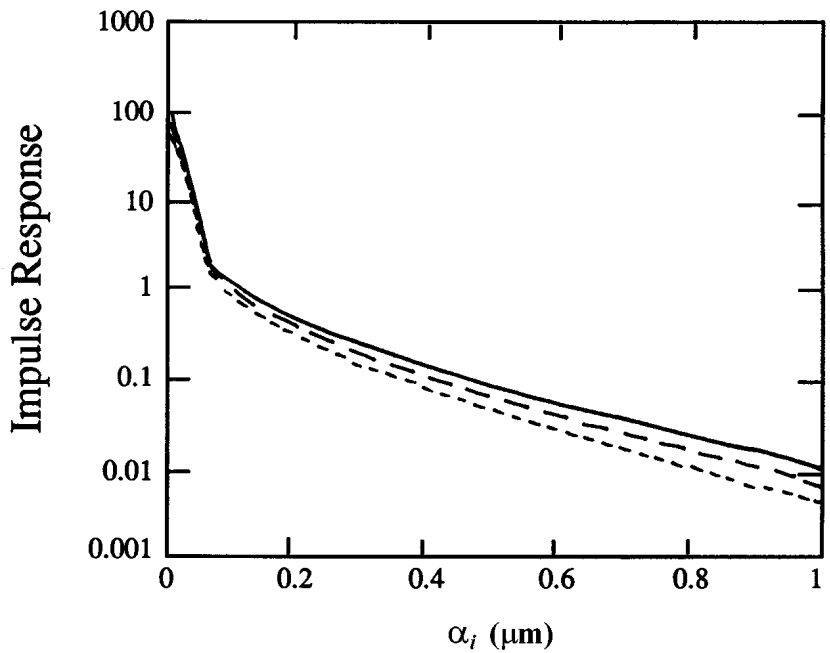

(b)

Fig. 3. Incoherent spatial impulse response [(a) outer segment, (b) inner segment] for three wavelengths: solid curve, $\lambda_{r}=680 \mathrm{~nm}$; short-dashed curve, $\lambda_{g}=550 \mathrm{~nm}$; long-dashed curve, $\lambda_{b}=460 \mathrm{~nm}$ (logarithmic scale for $y$ axis). 


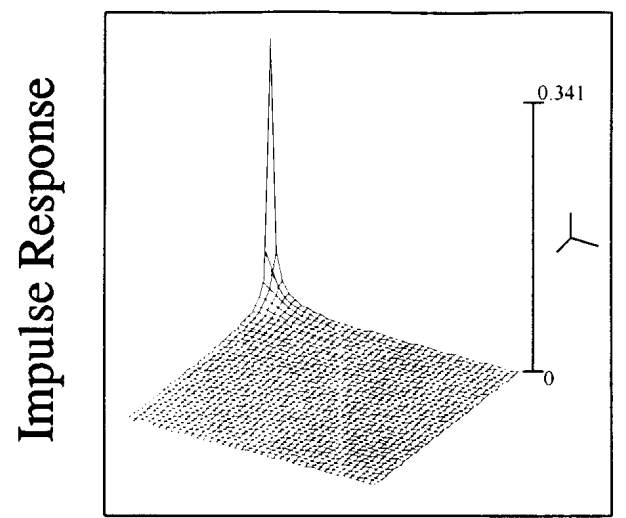

(a)

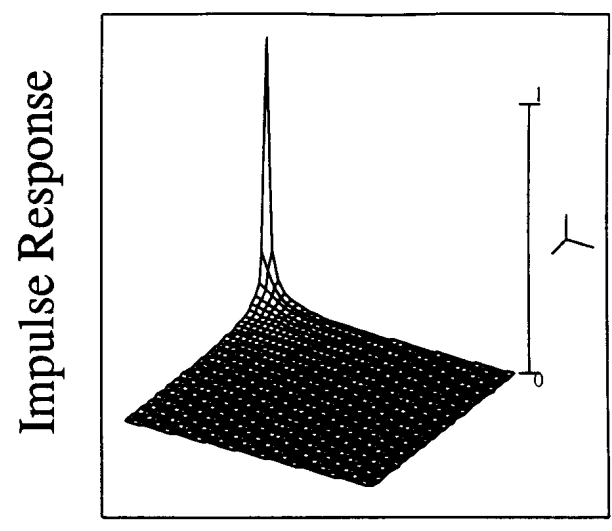

(b)

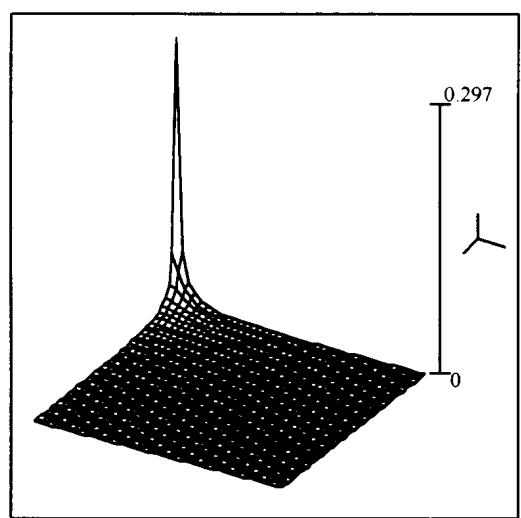

(c)

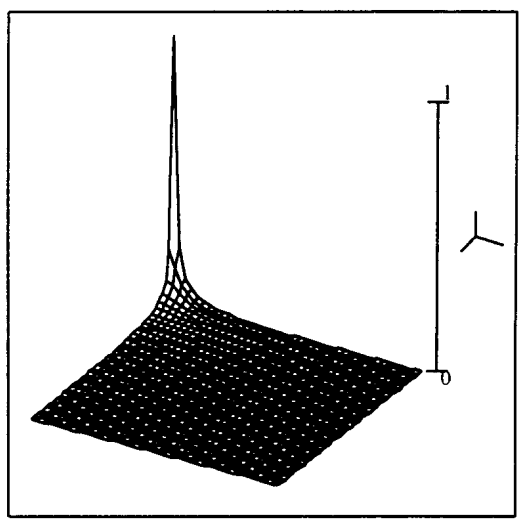

(d) $r(\mu \mathrm{m})$

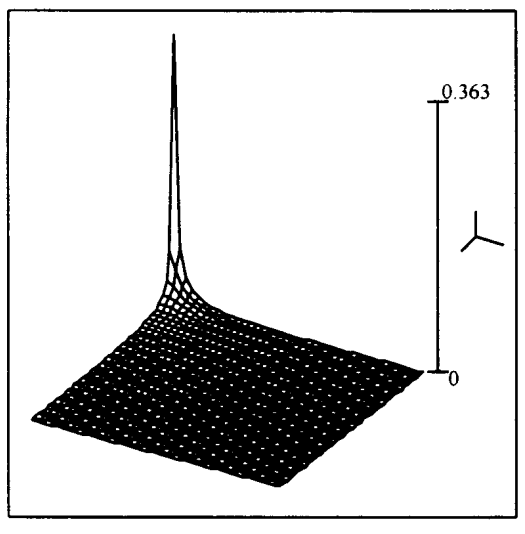

(e)

$r(\mu \mathrm{m})$

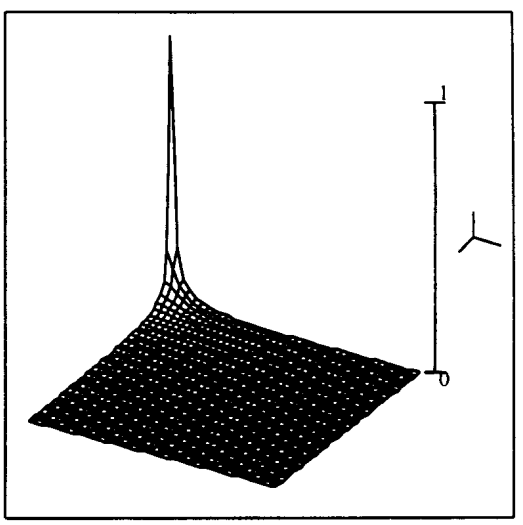

(f)

$\mathbf{r}(\mu \mathrm{m})$

Fig. 4. Three-dimensional representation of the three impulse responses shown in Fig. $3 . \quad$ (a), (b): $\quad \lambda_{r}=680 \mathrm{~nm} ;(\mathrm{c}),(\mathrm{d}): \quad \lambda_{g}=$ $550 \mathrm{~nm}$; (e), (f): $\lambda_{b}=420 \mathrm{~nm}$. Spatial coordinate: $r=\left|\mathbf{x}-\mathbf{x}^{\prime}\right|$ in micrometers.

refractive index of the waveguide; and $\beta_{0}$ is the modulus of the wave vector inside the waveguide.

If we fix the wavelength, then the range of the definition for $\beta_{0}$ is

$$
k n_{c} \leq \beta_{0, i} \leq k n_{i}
$$

with $n_{i}$ being the refractive index for the inner segment. An analogous condition holds for the outer segment.

In the present case, for the outer segment we calculate the following values: $\chi_{o, 0}=4.5 \times 10^{3} \mathrm{~mm}^{-1}$ for an average wavelength $\lambda_{r}=680 \mathrm{~nm} ; \chi_{o, 0}=5.6 \times 10^{3} \mathrm{~mm}^{-1}$ for $\lambda_{g}=550 \mathrm{~nm}$; and $7.4 \times 10^{3} \mathrm{~mm}^{-1}$ for the blue wavelength $\lambda_{b}=420 \mathrm{~nm}$. Similarly, for the inner segment, we calculate $\chi_{0, i}=1.7 \times 10^{3} \mathrm{~mm}^{-1}$ for $\lambda_{r}$, and $1.68 \times 10^{3} \mathrm{~mm}^{-1}$ and $2.8 \times 10^{3} \mathrm{~mm}^{-1}$ for $\lambda_{g}$ and $\lambda_{b}$, respectively. To define the interval $\left(\mathbf{x}-\mathbf{x}^{\prime}\right)$ [see relations (25) and (26)] we assume that the maximum difference cannot exceed the total diameter of each segment. In Fig. 3 we show the one-dimensional impulse response of the two segments for the three wavelengths under consideration (a logarithmic scale has been used for the $y$ axis). We observe that the functions are close to a Gaussian function with a small bandwidth and that for points $>0.8 \mu \mathrm{m}$ the response is almost negligible, demonstrating that the signal is packed near the waveguide axis. This behavior is similar for the three wavelengths in the inner segment, whereas in the outer segment the bandwidth decreases from $\lambda_{r}$ (maximum bandwidth $\approx 1.0 \mu \mathrm{m}$ ) to $\lambda_{b}$ (maximum bandwidth $\approx 0.8 \mu \mathrm{m})$. The inner segment seems to transmit a higher percentage of luminous signal (approximately $70 \%$ more than in the outer segment).

We have represented the impulse response as a twodimensional function. All the results are shown in Fig. 4 for the three wavelengths $\lambda_{r}, \lambda_{g}$, and $\lambda_{b}$ under consideration. The similar behavior that we notice in all three cases (the values for the outer and the inner segments are normalized with respect to the maximum value of the inner segment) demonstrates that the photoreceptor behaves as a low-pass filter.

Using Eq. (26), we numerically analyzed the transfer function for the three wavelength values and for fixed modal parameters $\chi_{0, o}$ and $\chi_{0, i}$. The results are given in Fig. 5. The cutoff frequency is inversely proportional to the wavelength for a fixed angular aperture of the photoreceptor. The outer segment exhibits a wider spatialfrequency range, and it seems to act as a high-pass filter. This effect is more noticeable for the red wavelength. Also, for all the frequencies, the outer segment is processing a higher percentage of energy than the inner segment (approximately an average of 30\% more). The total spectrum represented in Figs. 5(b), 5(d), and 5(f), respectively, behaves much like that of the inner segment. The final effect is a reduced-range low-pass filter. As in the previous numerical example, this result is more appreciable for the red wavelength. The spatial resolution 
of the photoreceptor is determined by the spatial resolution of the inner segment.

Because the transfer function depends on the modal parameter, we analyzed its behavior for fixed spatial frequency. The results are shown in Fig. 6 (top row, outer segment; bottom row, inner segment; a logarithmic scale was used for the $y$ axis). The range of values for the modal parameters given in inverse millimeters was calculated as follows. For $\lambda_{r}=0.68 \mu \mathrm{m}, 676 \leq \chi_{o, 0} \leq$ $4600 \mathrm{~mm}^{-1}$ (outer segment) and $676 \leq \chi_{i, 0} \leq 1700 \mathrm{~mm}^{-1}$ (inner segment). For $\lambda_{g}=0.55 \mu \mathrm{m}, 240 \leq \chi_{o, 0} \leq$ $5725 \mathrm{~mm}^{-1}$ (outer segment) and $240 \leq \chi_{i, 0} \leq 2160 \mathrm{~mm}^{-1}$ (inner segment). For $\lambda_{b}, 380 \leq \chi_{o, 0} \leq 7460 \mathrm{~mm}^{-1}$ (outer segment) and $380 \leq \chi_{i, 0} \leq 2790 \mathrm{~mm}^{-1}$ (inner segment). The total function would be obtained by representation of both functions jointly (results not shown). These func-

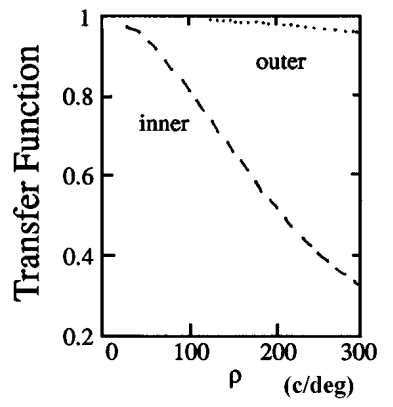

(a)

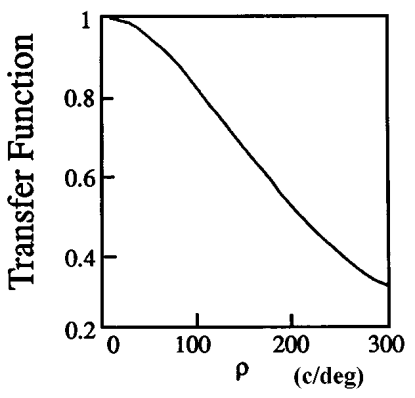

(b)

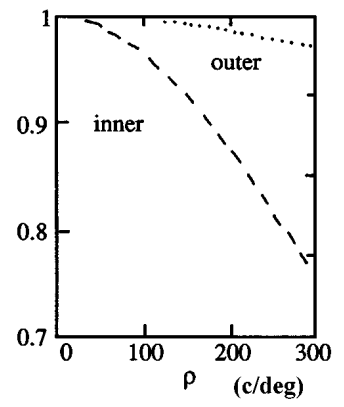

(c)

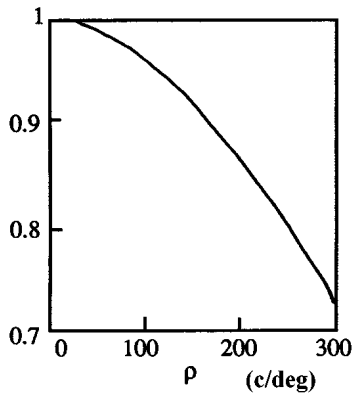

(d)

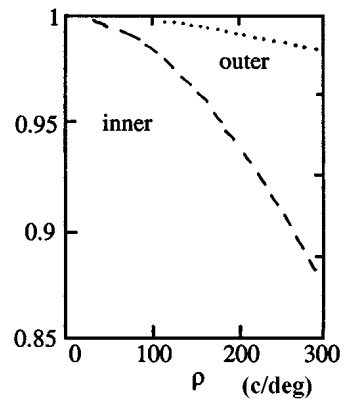

(e)

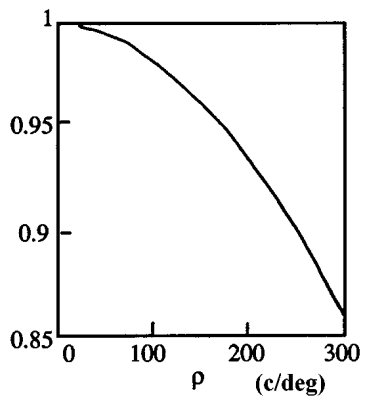

(f)

Fig. 5. Transfer functions of the inner and the outer segments (top row) and total function (bottom row) for the three wavelengths (values same as in Fig. 4). (a), (b): $\lambda_{r} ;$ (c), (d): $\lambda_{g}$; (e), (f): $\lambda_{b}$. The values of the modal parameters are fixed (see text for details). Dashed curve, inner segment; dotted curve, outer segment. c/deg stands for cycles per degree.

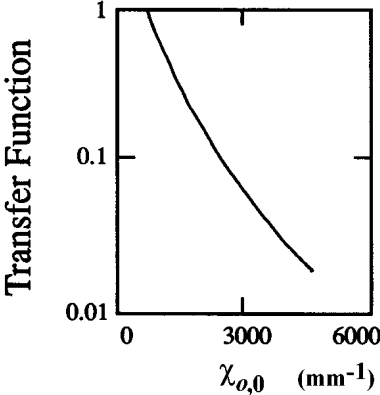

(a)

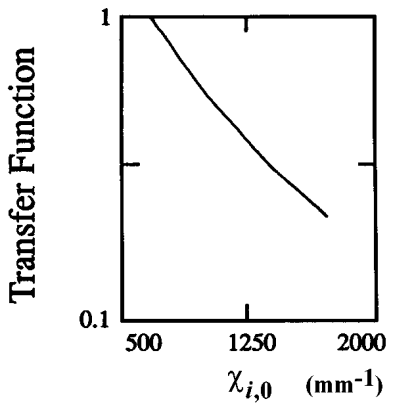

(b)

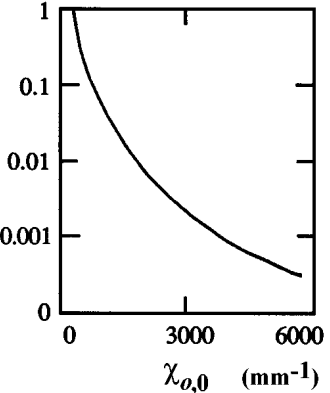

(c)

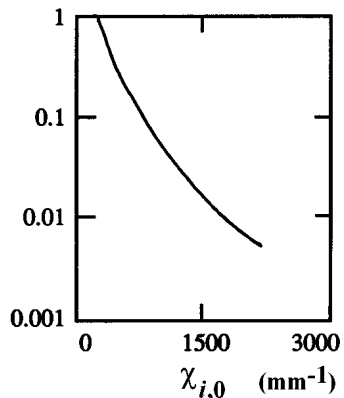

(d)

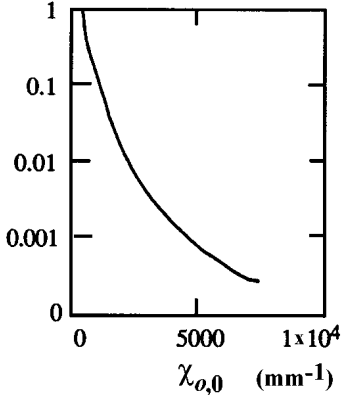

(e)

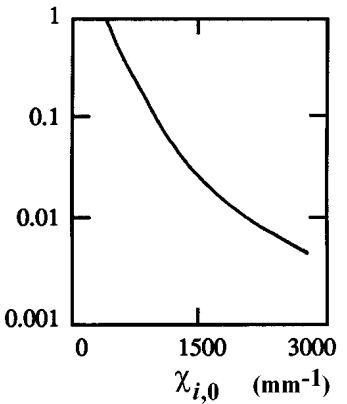

(f)

Fig. 6. Transfer function versus modal parameter $\chi_{0}$. Values for fixed cutoff frequency: (a), (b): $\lambda_{r}, 500 \mathrm{~mm}^{-1} ;(\mathrm{c}),(\mathrm{d}): \lambda_{g}$, $140 \mathrm{~mm}^{-1}$; (e), (f): $\lambda_{b}, 120 \mathrm{~mm}^{-1}$. Top row: outer segment; bottom row: inner segment (logarithmic scale for $y$ axis). 
tions overlap for the entire inner segment range (smaller), and hence the total variation is closely related to that of the outer segment. Note, also, that for the red wavelength, $\lambda_{r}$, the overlap part of both functions is smaller than in the case for the $\lambda_{g}$ and the $\lambda_{b}$ wavelengths. Depending on the modal parameters, these total transfer function distributions indicate how the incident signal creates channels with different percentages of energy as it propagates from the lower bound of the modal parameter to the upper one (assuming a mechanism by which these channels could be created by a selective excitation of modal orders). This effect requires further investigation. It can also be noted that for the blue wavelength, $\lambda_{b}$, a wider range of modal-parameter values is permitted, so that both the inner and the outer segments cover an equivalent range of values for the parameters $\chi_{0}$.

\section{DISCUSSION AND CONCLUSIONS}

Based on a formalism for the initial field striking the aperture pupil of a photoreceptor, we have presented a generalization, in terms of two integrals, to the case of a receptor with variable cross section. The receptor is represented by two sections: the inner and the outer segments, for which the boundary conditions have been established. Developing the analogies of this formulation with the response of a linear space-invariant optical system (homogeneous receptor), we have introduced an expression for the incoherent spatial impulse response in terms of a Kelvin function. According to the behavior of linear invariant systems, the Fourier transform of the impulse response is the transfer function, and this function is derived for both the inner and the outer segments. This function depends on both the modal parameter defining the waveguiding regime for the segment and the spatial frequency. A numerical analysis leads to some specific conclusions in the frequency domain of the behavior of the photoreceptor. Both the inner and the outer segments behave like low-pass filters, although for the outer segment a wider frequency range is obtained, particularly for the red wavelength. The cutoff frequency is inversely proportional to the wavelength value for a fixed angular aperture. Also, for a fixed spatial-frequency value, the transfer function depends on the modal parameters defining the number of modes confined in each segment. From these results one is allowed to conjecture that different channels of energy are created for each frequency as modes are excited from the lower to the upper bound of the modal parameter. Some differences are observed for three different wavelength values. Receptors transmitting blue wavelengths exhibit similar behavior for both segments, which implies that a similar number of channels of energy associated with modal parameters will be created. In contrast, for the red wavelength, receptors would exhibit a much more noticeable difference between the inner and the outer segments. These conjectures could lead to some other interesting conclusions regarding variable-cross-section receptors. Also, because the total impulse response is known, the calculation of the autocorrelation and the power spectrum, for both coherent and incoherent illumination, and the definition of particular output signals will be the subject of future research.

\section{REFERENCES AND NOTES}

1. J. M. Enoch, "Visualization of waveguide modes in retinal receptors," Am. J. Ophthalmol. 51, 1107-1118 (1961).

2. For a general recent review of the Stiles-Crawford effect and modal patterns in photoreceptors, see J. M. Enoch and V. Lakshminarayanan, "Retinal fiber optics," in Visual Optics and Instrumentation, Vol. 1 of Vision and Visual Dysfunction, W. N. Charman, ed. (Macmillan, London, 1991), pp. 280-310. A waveguide model of the StilesCrawford effect is given by A. Snyder and C. Pask, "The Stiles-Crawford effect: explanation and consequences," Vision Res. 13, 1115-1137 (1973).

3. J. M. Enoch and F. L. Tobey, Jr., eds., Vertebrate Photoreceptor Optics (Springer-Verlag, Berlin, 1981), and references therein.

4. C. Pask and K. F. Barrell, "Photoreceptor optics. I. Introduction to formalism and excitation in a lens-photoreceptor system," Biol. Cybern. 36, 1-8 (1980).

5. R. F. Alverez-Estrada and M. L. Calvo, "Neutron fibres: a possible application of neutron optics," J. Phys. D 17, 475-502 (1984).

6. M. L. Calvo and V. Lakshminarayanan, "Initial field and energy flux in absorbing optical waveguides. I. Theoretical formalism,” J. Opt. Soc. Am. A 4, 1037-1042 (1987).

7. V. Lakshminarayanan and M. L. Calvo, "Initial field and energy flux in absorbing optical waveguides. II. Implications," J. Opt. Soc. Am. A 4, 2133-2140 (1987).

8. M. L. Calvo and V. Lakshminarayanan, "An analysis of the modal field in absorbing optical waveguides and some useful approximations," J. Phys. D 22, 603-610 (1989).

9. J. W. Goodman, "Linear space-variant optical data processing," in Optical Information Processing Fundamentals, Vol. 48 of Topics in Applied Physics, S. H. Lee, ed. (SpringerVerlag, Berlin, 1981), Chap. 6.

10. M. L. Calvo, "Linear behavior in the aperture pupil of single photoreceptors: consequences related to the degree of inhomogeneity,” Biol. Cybern. 54, 201-210 (1986).

11. M. L. Calvo, "Some physical features on the mechanism of vision and photoreceptors," in Laser and Ultrafast Phenomena, A. Piskarskas, ed. (Vilnius U. Press, Vilnius, Lithuania, 1991), Vol. 4, pp. 167-174.

12. R. L. De Valois and K. K. De Valois, Spatial Vision, Vol. 14 of Oxford Psychology Series (Oxford U. Press, New York, 1988), Chap. 6.

13. A. Stacey and C. Pask, "Spatial-frequency response of a photoreceptor and its wavelength dependence. I. Coherent sources,” J. Opt. Soc. Am. A 11,1193-1198 (1994).

14. M. Abramowitz and I. Stegun, Handbook of Mathematical Functions, 7th ed. (Dover, New York, 1968), p. 364.

15. A. W. Lohman and D. P. Paris, "Space-variant image formation," J. Opt. Soc. Am. 55, 1007-1013 (1965).

16. B. R. Horowitz, "Theoretical considerations of the retinal receptors as a waveguide," in Vertebrate Photoreceptor Optics, J. M. Enoch and F. L. Tobey, Jr., eds. (Springer-Verlag, Berlin, 1981), pp. 219-294.

17. R. L. Sidman, "The structure and concentration of solids in photoreceptor cells studied by refractometry and interference microscopy,” J. Biophys. Biochem. Cytol. 3, 15-30 (1957).

18. M. L. Calvo and P. K. Mondal, "Effects induced on the transverse spatial impulse response of an inhomogeneous photoreceptor with a nonsymmetric refractive index profile and arbitrarily located origin," Nuovo Cimento 9D, 261-273 (1987).

19. R. N. Bracewell, The Fourier Transform and Its Applications, (McGraw-Hill, New York, 1978), p. 422.

20. B. Borwein, D. Borwein, J. Medeiros, and J. McGowan, “The ultra structure of monkey foveal photoreceptors, with special reference to the structure, shape, size and spacing of the foveal cones," Am. J. Anat. 159, 125-146 (1980).

21. A. Snyder and J. D. Love, Optical Waveguide Theory (Chapman and Hall, London, 1983), p. 227. 